\title{
Clinical and Radiological Assessment of Post-Surgical Sequelae in Donor Site after Microsurgical Fibula Free Flap
}

\author{
Claudia Celotti ${ }^{1 *}$ (D), Sonia Herrero ${ }^{1}$, Marta Echevarría ${ }^{2}$, Paula Buded ${ }^{3}$, Manuel De Pedro ${ }^{1}$ and Farzin \\ Falahat $^{1}$
}

${ }^{* 1}$ Oral and Maxillofacial Department, Hospital Clínico San Carlos, Madrid, Spain

${ }^{2}$ Orthopedics and Traumatology Department, Hospital Clínico San Carlos, Madrid, Spain

${ }^{3}$ Complutense University, Madrid, Spain

\begin{abstract}
Objectives: Fibula free flap is commonly used in mandibular reconstruction. It is globally accepted that the morbidity in the donor site is minimal. The aim of this study was to assess the clinical and radiological morbidity of the donor-site after microsurgical fibula free flap.

Study design: A retrospective case series study was performed for clinical and radiological assessment of the donor-site in 18 patients who underwent oromandibular reconstruction with fibula free flap between the years 2008 and 2018 in Hospital Clínico San Carlos. Post-operative complications, and clinical and radiological variables were evaluated.

Results: The most prevalent clinical variables were cold intolerance in 11 patients (61.11\%), motor weakness in 10 patients $(55.56 \%)$, gait disorders in 9 patients $(50 \%)$ and sensory deficit in 9 patients $(50 \%)$. The main radiological complications were: knee arthrosis in 17 patients (94.44\%), remaining fibula distal ends osteoporosis in 14 patients (77.78\%) and ankle arthrosis in 13 patients (77.78\%). A statistically significant relation was found between distal fibula remnant length $<80$ $\mathrm{mm}$ and: Gait disorders, instability, motor weakness and distal fibula remnant osteoporosis.

Conclusions: It is difficult to assess the morbidity in the donor-site due to subjectivity and inter individual variability of symptoms. The results showed a low complication rate, however, long term effects may include an alteration in the lower limb functionality.
\end{abstract}

Keywords

Morbidity, Donor-site, Fibula free flap, Mandibular reconstruction

\section{Introduction}

Fibula free flap is probably the most employed one in oromandibular reconstruction. It was first described by Taylor, et al. [1] in 1975 in a tibial defect reconstruction. Its implementation for orofacial reconstruction was not described until 1989 by Hidalgo [2] in the mandibular reconstruction of 12 osseous defects.

It is a free microvascular flap that provides not only bone but also skin, fascia and/or muscle for reconstruction. It is useful for reconstruction of defects greater than $14 \mathrm{~cm}$, it can be remodelled employing multiple osteotomies, it allows prompt dental implant placement and it is associated with low morbidity of the donor-site [3]. Immediate postoperative complications include bleeding, hemodynamic instability and venous tromboembolism, while late complications involve non-union of the transplanted bone, hematoma and recipient site infection. Regarding donor site, the most highlighted complications are limb chronic pain, muscle fatigue, edema, foot drop, anesthesia in the anterolateral region of the leg (caused by common peroneal nerve injury), ankle instability, rear foot valgus and claw toe [4-6]. It is globally accepted that the morbidity in the donor site is minimal, however, there is few literature on the subject.

The purpose of our study is to evaluate from a clinical and radiological point of view the morbidity of the donor site after microsurgical fibula free flap in order to estimate the

*Corresponding author: Dr. Claudia Celotti, Resident, Oral and Maxillofacial Department, Hospital Clínico San Carlos, C/ Alejandro Villegas $57,1^{\circ}, 28043$, Madrid, Spain, Tel: +34-658-249-801

Accepted: August 08, 2020

Published online: August 10, 2020

Citation: Celotti C, Herrero S, Echevarría M, et al. (2020) Clinical and Radiological Assessment of Post-Surgical Sequelae in Donor Site after Microsurgical Fibula Free Flap. J Head Neck Surg 2(1):54-59 
Citation: Celotti C, Herrero S, Echevarría M, et al. (2020) Clinical and Radiological Assessment of Post-Surgical Sequelae in Donor Site after Microsurgical Fibula Free Flap. J Head Neck Surg 2(1):54-59

incidence of complications and establish a hypothesis about possible causal factors.

\section{Materials and Methods}

\section{Study design}

A descriptive, observational retrospective case series study was performed for clinical and radiological assessment of the donor-site in all consecutive patients who underwent oromandibular reconstruction with microsurgical fibula free flap between the years 2008 and 2018 in the Oral and Maxillofacial Service of Hospital Clínico San Carlos (Madrid, Spain).

\section{Study variables}

The recorded clinical variables were: Hypoesthesia, chronic pain, motor fatigue, gait alterations, instability, foot edema, cold intolerance, claw toe, weak dorsiflexion of the great toe, plantar flexion deficit, lower limb length discrepancy, subjective valgus and subjective satisfaction degree (good, average, poor).

Immediate post-operative variables included compartimental syndrome, thromboembolic complications in lower limbs and surgical donor-site infection. Donor lower limb function was quantified using Takakura scale for ankle arthrosis [7,8] and Kellgren [9] and Ahlbäck [10] scales for knee arthrosis.

Regarding radiological variables, these include: resected graft length $(\mathrm{mm})$, distal and proximal fibula remnant length $(\mathrm{mm})$, fibula osteoporosis, heterotopic ossification, tibiofibular radiological space, tibiofibular radiological overlap, spontaneous fractures and proximal fibula migration.

Rearfoot valgus was quantified by measuring lateral distal tibial angle (LDTA), considering an angle $<86^{\circ}$ as valgus (the published range for LDTA is $89 \pm 3^{\circ}$ ).

Independent variables, such as demographic characteristics and potential risk factors, were also recorded (Table 1).

\section{Data collection}

The assessment period comprised February 2019 to June 2019. Informed Consent was obtained and a "Patient Information Sheet" was handed to all patients included in the study. Data was obtained by: Anatomical and functional explorations of lower limbs; a telephone questionnaire for subjective examination of sensitivity, pain, functional capacity and aesthetic result; postero-anterior and lateral X-ray and photographs of both lower limbs. Data collection was completed by patient's medical history data and operative reports, according to our Hospital Science Investigation/Communication protocols.

In all patients, anatomical and functional examination and $\mathrm{X}$-ray analysis were conducted by the same person, who was foreign to all surgical procedure.

\section{Statistical analysis}

Descriptive analyses: Qualitative variables were summarized by their frequency distribution as well as quantita-
Table 1: Patient's variables.

\begin{tabular}{|c|c|c|}
\hline \multicolumn{2}{|c|}{ Demographic and surgical variables } & \multirow{2}{*}{$\begin{array}{l}\mathbf{N}=18 \mathbf{n}(\%) \\
8(44.4)\end{array}$} \\
\hline Age & $<60$-years-old & \\
\hline & $>60$-years-old & $10(55.6)$ \\
\hline \multirow[t]{2}{*}{ Sex } & Male & $13(72.2)$ \\
\hline & Female & $5(27.8)$ \\
\hline \multirow[t]{2}{*}{ Cause of reconstruction } & $\begin{array}{l}\text { Squamous cell } \\
\text { carcinoma }\end{array}$ & $15(83.3)$ \\
\hline & Others & $3(16.7)$ \\
\hline \multicolumn{2}{|l|}{ Chemotherapy } & $7(38.89)$ \\
\hline \multicolumn{2}{|l|}{ Physical activity } & $7(38.89)$ \\
\hline \multirow[t]{2}{*}{ Woundclosure } & Pimary closure & $10(55.6)$ \\
\hline & Free skin graft & $8(44.4)$ \\
\hline \multicolumn{3}{|l|}{ Clinical variables } \\
\hline \multicolumn{2}{|l|}{ Cold intolerance } & $11(61.1)$ \\
\hline \multicolumn{2}{|l|}{ Motor weakness } & $10(55.6)$ \\
\hline \multicolumn{2}{|l|}{ Sensitive deficit } & $9(50)$ \\
\hline \multicolumn{2}{|l|}{ Gait disorders } & $9(50)$ \\
\hline \multicolumn{2}{|l|}{ Chronic pain } & $8(44.4)$ \\
\hline \multicolumn{2}{|l|}{ Hallux claw toe } & $6(33.3)$ \\
\hline \multicolumn{2}{|c|}{ Weak dorsiflexion of the great toe } & $6(33.3)$ \\
\hline \multicolumn{2}{|l|}{ Instability } & $5(27.8)$ \\
\hline \multicolumn{2}{|l|}{ Subjective valgus } & $5(27.8)$ \\
\hline \multicolumn{2}{|l|}{ Plantar flexion deficit } & $4(22.2)$ \\
\hline \multicolumn{2}{|l|}{ Foot edema } & $3(16.7)$ \\
\hline \multicolumn{2}{|c|}{ Lower limbs length discrepancy } & $1(5.6)$ \\
\hline \multicolumn{3}{|l|}{ Radiological variables } \\
\hline \multicolumn{2}{|l|}{ Knee arthrosis } & $17(94.4)$ \\
\hline \multicolumn{2}{|l|}{ Ankle osteoporosis } & $14(77.8)$ \\
\hline \multicolumn{2}{|l|}{ Ankle arthrosis } & $13(72.2)$ \\
\hline \multicolumn{2}{|l|}{ Ipsilateral tibial deformity } & $4(22.2)$ \\
\hline \multicolumn{2}{|l|}{ Heterotopic ossification } & $2(11.1)$ \\
\hline \multicolumn{2}{|c|}{ Ipsilateral tibial spontaneous fractures } & $0(0)$ \\
\hline \multicolumn{2}{|l|}{ Proximal fibula migration } & $0(0)$ \\
\hline
\end{tabular}

tive variables by their mean and standard deviation ( \pm SD). The continuous non-normally distributed variables were summarized by the median and interquartile range (IQR = P25-P75). Comparison between groups: In case of qualitative variables, comparison was evaluated by the test of Chisquared, or by Fisher's exact test in case more than $25 \%$ of the expected values were less than five. To compare between the two groups, the non-parametric Mann-Whitney test for quantitative variables were used due to the small size. The null hypothesis was rejected in each statistical test when $p<$ 0.05 . Analysis was performed using IBM SPSS Statistics v21 software.

\section{Ethical considerations}

The present study was approved by the Hospital's Clinical 
Citation: Celotti C, Herrero S, Echevarría M, et al. (2020) Clinical and Radiological Assessment of Post-Surgical Sequelae in Donor Site after Microsurgical Fibula Free Flap. J Head Neck Surg 2(1):54-59

Research Ethics Committee. We assure data confidentiality according to the Spanish Data Protection Regulation (Ley Organica de Protección de Datos y Carácter Personal 15/1999 and Reglamento general de protección de datos 2016/679).

The authors declare that there is no economical, professional or any other personal interest that could interfere the development of the present study.

\section{Results}

A total of 48 patients underwent oromandibular reconstruction with microsurgical fibula free flap in our Hospital between 2008 and 2018, only eighteen of which met inclusion criteria for the study. Thirty patients were excluded: 17 patients had died, 11 refused to participate and 2 could not be contacted.

The group consisted of 13 men (72.2\%) and 5 women (27.8\%), relation 2, 6:1 with predominance of men. At the time of operation, the mean age was 54 years (SD $=14.5$ ), with the oldest patient being 72-years-old and the youngest patient being 29-years-old. The median follow-up was 51.5 $(I Q R=18.0-98.7)$ months.

Mandibular resection was performed in 15 patients (83.3\%) with squamous cell carcinoma and in 3 patients (16.7\%) for other reasons. The mean length of the harvested grafts was $213.1 \mathrm{~mm}$ (SD = 27.2). Regarding proximal fibula remnant length, a mean of $84.5 \mathrm{~mm}(S D=16.8)$ was preserved (range $57 \mathrm{~mm}$ minimum and $134.4 \mathrm{~mm}$ maximum preserved), while distally, a mean fibula length of $86.8 \mathrm{~mm}$ ( $\mathrm{SD}=13.7$ ) was preserved $(66 \mathrm{~mm}$ as minimum and $117 \mathrm{~mm}$ as maximum preserved).
Tumor resection and simultaneous reconstruction surgery was performed in all patients (100\%). In 8 cases (44.4\%) a free skin graft was placed in donor site and in 10 cases (55.6\%) primary closure was achieved.

\section{Clinical complications}

Post-operative complications are shown in Table 1 . The main clinical sequelae found in our study were, in order of frequency: Cold intolerance in a total of 11 patients (61.1\%), motor weakness in 10 patients (55.6\%), gait disorders in 9 patients (50\%) and sensory deficit in 9 patients (50\%). Other complications include chronic pain in donor limb in 8 patients (44.4\%), weak dorsiflexion of the great toe in 6 cases $(33.3 \%)$ and claw toe also in 6 cases (33.3\%).

In relation to adjuvant chemotherapy, our study reveals a higher frequency of sensory deficit $(71.4 \%)$ and cold intolerance $(85.7 \%)$ in patients that received chemotherapy, although no statistical significance was found (Table 2).

Regarding surgical closure, primary closure showed a greater incidence of sensory deficit (60\%) and foot edema (30\%) compared to free skin graft placement, despite the fact no statistical significance was observed (Table 3).

The relationship between variables and the distal fibula remnant length preserved is shown in (Table 4). In our study, we found a statistically significant relationship between distal remnant length shorter than $80 \mathrm{~mm}$ and: Gait alteration $(p=0.015)$, instability $(p=0.036)$, and motor weakness $(p=$ 0.005).

Aesthetic outcome was rated as good by 10 patients (55.6\%) as average by 8 (44.4\%) and nobody considered a poor result.

Table 2: Relationship between variables and chemotherapy.

\begin{tabular}{|l|l|l|l|}
\hline \multirow{2}{*}{} & \multicolumn{2}{l|}{ Chemotherapy } & No \\
\cline { 2 - 4 } & Yes & P $=11$ & \\
\hline Sensitive deficit & $\mathrm{N}=7$ & $\mathrm{n}(\%)$ & \\
\hline Cold intolerance & $\mathrm{n}(\%)$ & $4(36.4)$ & 0.336 \\
\hline Distal fibula osteoporosis & $5(71.4)$ & $5(45.4)$ & 0.2254 \\
\hline
\end{tabular}

Table 3: Relationship between variables and modality of wound closure.

\begin{tabular}{|l|l|l|l|}
\hline & Free skin graft & Primary closure & P \\
& $\mathrm{N}=8$ & $\mathrm{~N}=10$ \\
$\mathrm{n}(\%)$ & $\mathrm{n}(\%)$ & 0.6353 \\
\hline Sensitive deficit & $3(37.5)$ & $6(60)$ & \\
\hline Foot edema & $0(0)$ & $3(30)$ & 1.000 \\
\hline Cold intolerance & $5(62.5)$ & $6(60)$ & $1(10)$ \\
\hline Compartimental syndrome & $2(25)$ & $1(10)$ & \\
\hline Surgical donor-site infection & $2(25)$ & $0(0)$ & \\
\hline Thromboembolic complications & $1(12.5)$ & & \\
\hline
\end{tabular}


Citation: Celotti C, Herrero S, Echevarría M, et al. (2020) Clinical and Radiological Assessment of Post-Surgical Sequelae in Donor Site after Microsurgical Fibula Free Flap. J Head Neck Surg 2(1):54-59

Table 4: Relationship between variables and distal fibula remnant.

\begin{tabular}{|c|c|c|c|}
\hline & \multicolumn{3}{|c|}{ Distal fibula remnant } \\
\hline & $\begin{array}{l}<80 \mathrm{~mm} \\
\mathrm{~N}=10 \\
\mathrm{n}(\%)\end{array}$ & $\begin{array}{l}\geq 80 \mathrm{~mm} \\
\mathrm{~N}=8 \\
\mathrm{n}(\%)\end{array}$ & $\mathbf{P}$ \\
\hline Gait alterations & $8(80)$ & $1(12.5)$ & 0.015 \\
\hline Instability & $5(50)$ & $0(0)$ & 0.036 \\
\hline Hallux claw toe & $5(50)$ & $1(12.5)$ & 0.2404 \\
\hline Motor weakness & $8(80)$ & $2(25)$ & 0.005 \\
\hline Plantar flexion deficit & $3(30)$ & $1(12.5)$ & 0.7513 \\
\hline $\begin{array}{l}\text { Weak dorsiflexion of the great } \\
\text { toe }\end{array}$ & $3(303)$ & $3(37.5)$ & 1.000 \\
\hline Subjective valgus & $1(10)$ & $4(50)$ & 0.176 \\
\hline $\begin{array}{l}\text { Objective valgus - lateral distal } \\
\text { tibial angle (LDTA) }<86^{\circ}\end{array}$ & $0(0)$ & $2(25)$ & \\
\hline Distal fibula osteoporosis & $10(100)$ & $50(4)$ & 0.023 \\
\hline Ankle arthrosis & $9(90)$ & $50(4)$ & 0.176 \\
\hline Foot edema & $3(30)$ & $0(0)$ & \\
\hline
\end{tabular}

Table 5: Relationship between variables and physical activity.

\begin{tabular}{|l|l|l|l|}
\hline & \multicolumn{3}{|l|}{ Physical activity } \\
\cline { 2 - 4 } & Yes & No & P \\
& $\mathrm{N}=7$ & $\mathrm{~N}=11$ & \\
& $\mathrm{n}(\%)$ & $\mathrm{n}(\%)$ & \\
\hline Ankle arthrosis & $4(57.1)$ & $9(81.8)$ & 0.326 \\
\hline Knee arthrosis & $6(85.7)$ & $11(100)$ & 0.389 \\
\hline Fibula osteoporosis & $4(57.1)$ & $10(90.9)$ & 0.245 \\
\hline Foot edema & $0(0)$ & $27.2(3)$ & \\
\hline
\end{tabular}

\section{Radiological complications}

Radiological variables are summarized in Table 1. In order of frequency, the most prevalent radiological finding was knee arthrosis in a total of 17 patients (94.4\%). 14 patients (77.8\%) presented osteoporosis in distal fibula remnant and 13 patients $(72.2 \%)$ ankle arthrosis. We also found, to a lesser extent, tibial deformation in 4 cases $(22.2 \%)$ and heterotopic ossification in 2 cases (11.1\%). The mean lateral distal tibial angle was $90.39^{\circ}$ and two patients (11.1\%) presented valgus, with a LDTA $<86^{\circ}$.

No statistical significance was observed between age and the next radiological variables: Ankle arthrosis, knee arthrosis, fibula osteoporosis and tibial deformity.

In regard of physical activity and sport practising, a higher incidence in ankle arthrosis (81.8\%) ( $p=0.326)$, knee arthrosis $(100 \%)$ ( $p=0.389)$ and distal fibula remnant osteoporosis $(90.9 \%)(p=0.245)$ was observed in patients that did not practise physical exercise during follow-up, compared to those patients who did (Table 5), although there was no statistically significant difference between both groups.

A statistical significant $(p=0.023)$ higher percentage of osteoporosis (100\%) was found in patients with distal fibula remnant length $<80 \mathrm{~mm}$ compared to those cases in which distal fibula remnant was longer than $80 \mathrm{~mm}$ (50\%) (Table 4).

When analysing Takakura scale for ankle arthrosis assessment, eight patients (44.4\%) presented grade 1 in the mentioned scale, four patients $(22.2 \%)$ grade 2 , one patient $(5.6 \%)$ grade 3 and five patients (27.8\%) did not present any grade of ankle arthrosis. We evaluated knee arthrosis degree employing two different scales, which are the Kellgren and Ahlbäck, scales. The results of Kellgren scale for knee arthrosis assessment were the following: 7 patients (38.9\%) presented grade 1 , five patients $(28.8 \%)$ grade 2 , also five patients $(28.8 \%)$ presented grade 3 and only one patient (5.6\%) did not present any grade of arthrosis. Ahlbäck scale assessment showed 12 patients $(66.7 \%)$ with grade 1 , five patients $(27.8 \%)$ with grade 2 and only one patient (5.6\%) with no arthrosis.

\section{Discussion}

Although many authors have studied the morbidity of the donor site after microsurgical fibula free flap, the real impact on the patient's functionality is currently unknown.

The most prevalent clinical complications in our study were cold intolerance in $61.1 \%$ of cases and sensory deficit in $50 \%$. Authors like Goodacre, et al. [11]. Reported cold intolerance in $44.4 \%$ of patients following microsurgical fibula free flap. Our series showed a higher percentage of sensory deficit (71.4\%) and cold intolerance $(85.7 \%)$ in patients that had received chemotherapy treatment, however, this relationship is not statistically significant. This fact could be explained by the neurotoxic effect of some antineoplastic agents, as described by Velasco R, et al. [12] in their article.

Motor weakness in $55.6 \%$ and gait alterations in $50 \%$ of the cases in our series also represent two of the main clinical sequelae that affect patient's functionality. Lee, et al. [13] in their study about donor site morbidity support a possible association between gait alteration and change in load transmission through the fibula, as fibula bears $1 / 6$ of total body weight and is part of the osteoligamentous complex of both knee and ankle joints $[6,14]$.

We also found chronic pain in $44.4 \%$ of patients in our study, a symptom described likewise by other authors such as J Farhadi, et al. in their study, [5] in which $30 \%$ of patients reported mild pain and $10 \%$ severe pain.

Other highlighted variables are weak dorsiflexion of the great toe and claw toe, both found in $33.3 \%$ of cases in our study. Sieg, et al. [15] described a frequency of $27 \%$ regarding claw toe. Lee, et al. [13] reported in their study an incidence of $30 \%$ in weakness of the long toe flexor and extensor muscles that could be explained, according to the authors, by peripheral nerve injury or muscle disinsertion occurred during surgery.

Some clinical complications mentioned in our study such as instability $(27.8 \%)$, subjective valgus $(27.8 \%)$, plantar flexion weakness $(22.2 \%)$, foot edema (16.7\%) and limb length discrepancy $(5.6 \%)$ are reflected in the published scientific literature, and studies like those by Ling, et al. [16] mention percentages similar to ours. 
The most noticeable radiological complication found in our study is distal fibula remnant osteoporosis, present in $77.8 \%$ of patients, a fact that concurs with Ajay Kumar Ver$m a ' s$, et al. [6] study, in which the mentioned finding was present in $76.5 \%$ of cases. The cited article also reports heterotopic ossification in $12.9 \%$ of the cases, results consistent with our series, with a reported rate of $11.1 \%$.

The most frequent radiological complication was knee arthrosis in $94.4 \%$ of our cases; nevertheless, we could not find related literature on the subject. Most patients presented grade 1 in both Ahlbäck and Kellgren scales. We found ankle arthrosis in $72.2 \%$ of patients, showing concordance between our series and J Farhadi's, et al. [5], where this finding appeared in $80 \%$ of their cases. Most patients presented grade 1 in Takakura scale.

Preserving the distal 70 to $80 \mathrm{~mm}$ of the fibula, including tibio-fibular syndesmosis is related to a lower number of complications, especially ankle instability [17]. Thereby, in our study a distal fibula remnant shorter than $80 \mathrm{~mm}$ is related to gait disorders, instability, motor weakness and fibula osteoporosis in a statistically significant way. In agreement with other studies such as Ajay Kumar Verma's, et al. [6] we did not find statistically significant. Association between distal remnant fibula length shorter than $70-80 \mathrm{~mm}$ and valgus. This association could be expected in infants, as reported in Liu's S, et al. [18] study, finding $20 \%$ of valgus in their series and only in pediatric age. Relation between distal fibula remnant and other variables has been one of the main objectives of our study and according to other authors like Ajay Kumar Verma, et al. [6] the presence of functional complications may be due to changes in load transmission through the limb and also to the loss of lateral ankle stability after surgery.

When comparing complications resulting from primary closure versus free skin graft placement in donor site, Shindo, et al. [19] reported in their study a higher incidence of complications when performing primary closure, probably due to a higher tension on the suture line than in free skin graft placement. In our study, we also observed a higher incidence of sensory deficit and foot edema in patients that underwent primary closure.

Finally, subjective assessment of surgery and aesthetic results was rated as good by $55.6 \%$ of patients and as average by $44.4 \%$. None of our patients rated surgery and its morbidity as poor result.

It is difficult to assess the morbidity in donor site when performing mandibular reconstruction due to the subjectivity and inter individual variability of symptoms. The results showed a low complication rate considering the benefits derived from mandibular reconstruction; nevertheless, it can result in long-term functional alterations that affect the patient's daily living. We must emphasize that donor site longterm follow-up is crucial to avoid sequelae that can be prevented providing effective patient education, rehabilitation or postoperative physiotherapy and orthopedic systems if needed.

\section{Declarations of Interest}

None.

\section{Funding}

This research did not receive any specific grant from funding agencies in the public, commercial, or not-for-profit sectors.

\section{References}

1. Taylor G, Watson N (1978) One-stage repair of compound leg defects with free, revascularized flaps of groin skin and iliac bone. Plast Reconstr Surg 61: 494-506.

2. Hidalgo DA (1989) Fibula free flap: A new method of mandible reconstruction. Plast Reconstr Surg 84: 71-79.

3. Dean Ferrer A, Cebrián Carretero J, García-Rozado González Á (2014) Reconstrucción ósea con colgajo libre de peroné. SECOM, 671-683.

4. Bumbasirevic M, Stevanovic M, Bumbasirevic V, et al. (2014) Free vascularised fibular grafts in orthopaedics. Int Orthop 38: 1277-1282.

5. Farhadi J, Valderrabano V, Kunz C, et al. (2007) Free fibula donor-sitemorbidity: Clinical and biomechanical analysis. Ann Plast Surg 58: 405-410.

6. Verma AK, Kushwaha NS, Saini A, et al. (2016) Retrospective analysis of donor site morbidity following partial fibular resection. International Journal of Contemporary Medical Research 3: 1571-1574.

7. Takakura Y, Tanaka Y, Kumai T, et al. (1995) Low tibial osteotomy for osteoarthritis of the ankle. Results of a new operation in 18 patients. J Bone Joint Surg Br 77: 50-54.

8. Tanaka Y, Takakura Y, Hayashi K, et al. (2006) Low tibial osteotomy for varus-type osteoarthritis of the ankle. J Bone Joint Surg $\mathrm{Br}$ 88: 909-913.

9. Kellgren JH, Lawrence JS (1957) Radiological assessment of osteo-arthrosis. Ann Rheum Dis 16: 494-502.

10. Ahlbäck S (1968) Osteoarthrosis of the knee. A radiographic investigation. Acta Radiol [Diagn] (Stockh) Suppl 277: 7-72.

11. Goodacre TE, Walker CJ, Jawad AS, et al. (1990) Donor site morbidity following osteocutaneous free fibula transfer. $\mathrm{Br} J$ Plast Surg 43: 410-412.

12. Velasco R, Bruna J (2010) Chemotherapy-induced peripheral neuropathy: An unresolved issue. Neurología 25: 116-131.

13. Lee EH, Goh JC, Helm R, et al. (1990) Donor site morbidity following resection of the fibula. J Bone Joint Surg Br 72: 129-131.

14. Rendenbach C, Rashad A, Hansen L, et al. (2017) Functional donor site morbidity longer than one year after fibula free flap: $A$ prospective biomechanical analysis. Microsurgery 38: 395-401.

15. Sieg P, Taner C, Hakim SG, et al. (2010) Long-term evaluation of donor site morbidity after free fibula transfer. $\mathrm{Br} J$ Oral Maxillofac Surg 48: 267-270.

16. Ling XF, Peng X (2012) What is the price to pay for a free fibula flap? A systematic review of donor-site morbidity following free fibula flap surgery. Plast Reconstr Surg 129: 657-674.

17. Wolff K, Ervens J, Herzog K, et al. (1996) Experience with the osteocutaneous fibula flap: Ananalysis of 24 consecutive re- 
Citation: Celotti C, Herrero S, Echevarría M, et al. (2020) Clinical and Radiological Assessment of Post-Surgical Sequelae in Donor Site after Microsurgical Fibula Free Flap. J Head Neck Surg 2(1):54-59

constructions of composite mandibular defects. Journal of Cranio-maxillofacial Surgery 24: 330-338.

18. Liu S, Tao S, Tan J, et al. (2018) Long-term follow-up of fibular graft for the reconstruction of bone defects. Medicine 97: e12605.

19. Shindo M, Fong BP, Funk GF, et al. (2000) The Fibula Osteocutaneous Flap in Head and Neck Reconstruction. Arch Otolaryngol Head Neck Surg 126: 1467-1472. 